\title{
MECHANICAL NATURE OF A SINGLE WALLED CARBON NANOTUBE USING LEGENDRE'S POLYNOMIALS
}

\author{
PARUL TIWARI \\ Centre for Advanced Computational Solutions, Lincoln University, New Zealand, and \\ Jaypee Institute of Information Technology, Noida, Uttar Pradesh, India; e-mail: parultiwari8922@gmail.com \\ PRIYANKA NAGAR \\ Jaypee Institute of Information Technology, Noida-201309, Uttar Pradesh, India
}

\begin{abstract}
This paper is concerned with the mechanical response of a single-walled carbon nanotube. Euler-Bernoulli's beam theory and Hamilton's principle are employed to derive the set of governing differential equations. An efficient variational method is used to determine the solution of the problem and Legendre's polynomials are used to define basis functions. Significance of using these polynomials is their orthonormal property as these shape functions convert mass and stiffness matrices either to zero or one. The impact of various parameters such as length, temperature and elastic medium on the buckling load is observed and the results are furnished in a uniform manner. The degree of accuracy of the obtained results is verified with the available literature, hence illustrates the validity of the applied method. Current findings show the usage of nanostructures in vast range of engineering applications. It is worth mentioning that completely new results are obtained that are in validation with the existing results reported in literature.
\end{abstract}

Keywords: Euler-Bernoulli's beam theory, nonlocal elasticity theory, Legendre's polynomial, aspect ratio, critical buckling load, Winkler and Pasternak elastic constants

\section{Introduction}

Nanotechnology has been continuously booming in the world wide research community for the last two decades as it offers numerous applications in the diversified field of biomaterials, communications, medicines and designing of efficient devices. Nanotubes, nanowires, nanotube resonators and nanoparticles are some nanomaterials in which beams and plates are widely used as main components at nano or micro length scale. One of the most fascinating elements in the periodic table is carbon that has several allotropes (diamond, fullerene, graphite, carbon nanotubes and graphene) according to its hybridization states. Carbon nanotubes were discovered by Iijima (1991) and formed by curling a graphitic sheet in a way which produced a class of materials that possess extraordinary mechanical and electrical properties (Dai et al., 1996) and (Kim and Lieber, 1999). These nanotubes are used as nanobeams in microelectromechanical and nanoelectromechanical systems ( $\mathrm{Li}$ et al., 2003). These nanotubes are stronger and stiffer than any other materials because of high elastic modulus of graphite sheets. Minuscule size plays a considerable role in the analysis of mechanical nature of these nanotubes and, thus, it is hard to ignore.

Atomistic modeling, nanoscale continuum mechanics and continuum mechanics are three main methods used to define mechanical properties of carbon nanotubes. In atomistic modeling, the position of atoms are computed based on their interactive forces and end conditions ( $\mathrm{Lu}$ and Bhattacharya, 2005). Atomistic modeling is very costly and time consuming. However, nano-scale continuum mechanics approach replaces the $\mathrm{C}-\mathrm{C}$ bond by a continuum element and can be 
used at nano-scale (Rafiee and Moghadam, 2014). Continuum mechanics states that the stress at each point of the body is a function of strain at the same point only. At nanoscale, size effects play a vital role in investigating the mechanical behavior as compared to that in macroscopic scale. But it is a well known fact that continuum beam theories are scale free, that is why these theories are not suitable for predicting small-scale effects of single walled carbon nanotubes. Therefore, to study the buckling behavior of carbon nanotubes the need for nonlocal continuum mechanics (Eringen, 1972, 1983) takes place. Non-classical (Nonlocal) elasticity theory has more information on forces between atoms than the classical elasticity theory. Thus it has been widely used for accurate and fast analysis of carbon nanotubes.

Initially, Peddieson et al. (2003) implemented nonlocal theory to nanotechnology. To investigate buckling behavior of carbon nanotubes, nonlocal continuum models were developed and used for general edge conditions by Wang Q. et al. (2006). Murmu and Pradhan (2009) employed a differential quadrature method alongwith the nonlocal Timoshenko beam theory. Challamel (2011) applied higher-order shear beam theories for buckling of nanobeams. Differential model of Eringen's theory is applied to formulate elastic beam theories by Reddy and El-Borgi (2014). Pradhan and Reddy (2011) used a differential transform technique to determine the buckling load of CNTs using different boundary conditions. Nagar and Tiwari (2017) applied successive differentiation approach to study characteristics of carbon nanobeams. Ansari et al. (2011) reported thermal buckling analysis of embedded single-walled carbon nanotube modeled through the Timoshenko beam model. Chirality effect was studied by Semmah et al. (2015) for zig-zag single walled carbon nanotubes. Chakraverty and Behera (2015) proposed a numerical technique to study vibration and buckling behavior of nanobeams using two types of elastic medium. Nejad and Hadi (2016) used theory of nonlocal elasticity to analyse bending behavior of bi-directional functionally graded beams. Norouzzadeh and Ansari (2017) predict the mechanical nature of nanobeams using the integral model of Eringen's theory. A review has been done for the modeling of carbon nanotubes using different models by Sakharova et al. (2017). Although several methods are used in literature to analyse the buckling behavior, authors find the importance of using the Rayleigh-Ritz method with Legendre's polynomials.

In the present paper, the buckling characteristics of single walled carbon nanotubes resting on a two-parameter elastic medium are analyzed using the Rayleigh-Ritz method, and Legendre's polynomials are used as shape functions. Nonlocal elasticity theory in conjunction with the Euler-Bernoulli beam theory (EBT) is used to obtain the governing equation of motion. A solution to the governing equation is obtained in the form of an eigen value problem for which a simple code is generated. Outputs of the problem are plotted graphically, and the interpretation of results is validated with those reported in literature (Wang C.M. et al., 2006). The effect of change in temperature is also observed for different room environments. The impact of distinct parameters on the lowest buckling load is examined and graphs are used to draw conclusions.

\section{Problem formulation}

According to nonlocal elasticity theory (Eringen, 2002), the stress at a specific point in the system is considered as a function of the strain state at all points of the system. The nonclassical stress tensor $\boldsymbol{\sigma}$ at a point $x$ over the volume $V$ is determined by the following relation

$$
\boldsymbol{\sigma}(x)=\int_{V} K(D, \xi) \boldsymbol{\tau} d V\left(x^{*}\right)
$$

where $\boldsymbol{\tau}$ is the local stress tensor, $K$ is a nonclassical modulus of two parameters $D$ and $\xi$. The nonlocal parameter is $\xi=\left(e_{0} a\right)^{2}$ in which $e_{0}$ is the material specific constant, $a$ is internal length parameter, $l$ is length of the tube and $D$ is Euclidean distance given as $\left|x^{*}-x\right|$. To solve 
integral constitutive relation (2.1), its simplified differential form is given by Eringen (1972) in the following form

$$
\left(1-\xi l^{2} \nabla^{2}\right) \boldsymbol{\sigma}(x)=\boldsymbol{\tau}(x)
$$

where $\nabla^{2}$ is the Laplace operator and $\boldsymbol{\tau}(x)$ is the local stress tensor defined by Hooke's law as follows

$$
\boldsymbol{\tau}(x)=\mathbf{C}(x): \varepsilon(x)
$$

where $\mathbf{C}(x)$ and $\varepsilon(x)$ is the elasticity tensor of the order four and the local strain tensor, respectively, and ":" denotes the double dot product. It is to be noted that in absence of $a$, equation (2.2) reduces to the constitutive equation of classical elasticity. Governing equations are formulated using the Eringen $(1972,1983)$ theory and EBT nonlocal theory of elasticity. For the exceptional strength and stiffness of carbon nanotubes, they are constructed with a very high length to diameter ratio, approximately 1, $32000000: 1$.

Hamilton's principle is expressed by the following relation

$$
\int_{0}^{t}\left[\left(\delta U_{s}+\delta U_{p}\right)-\delta U_{k}\right] d t=0
$$

where $\delta U_{s}, \delta U_{p}$ and $\delta U_{k}$ are the variations in strain, potential energy and kinetic energy, respectively.

The displacement components as stated in the Euler-Bernoulli beam theory are such that the transverse shear stress at the boundaries of the surface of the beam is zero and nonzero at other places. The displacement field is defined as

$$
u_{x}(x, z, t)=-z \frac{\partial u}{\partial x} \quad u_{y}(x, z, t)=0 \quad u_{z}(x, z, t)=u(x, t)
$$

where $u$ is the transverse deflection of a point of the beam in the mid plane. The non-zero strain displacement or bending strain is defined as

$$
\varepsilon_{x x}=-z \frac{\partial^{2} u}{\partial x^{2}}
$$

and the strain energy is

$$
U_{s}=\frac{1}{2} \int_{0}^{l} \int_{A} \sigma_{x x} \varepsilon_{x x} d A d x
$$

where $\sigma_{x x}$ is the normal stress, $l$ is the size of the beam and $A$ is the cross-sectional area.

Using (2.6) into (2.7), the strain energy is given as

$$
U_{s}=-\frac{1}{2} \int_{0}^{l} \int_{A} z \sigma_{x x} \frac{\partial^{2} u}{\partial x^{2}} d A d x=-\frac{1}{2} \int_{0}^{l} M \frac{\partial^{2} u}{\partial x^{2}} d x
$$

where $M=\int_{A} z \sigma_{x x} d A$ is defined as the bending moment. For free harmonic motion, the natural frequency $\omega$ of vibration is given by $u=u_{0}(x) \sin (\omega t)$. 
Therefore, the strain and kinetic energy of the beam are expressed as

$$
U_{s}=-\frac{1}{2} \int_{0}^{l} M \frac{d^{2} u_{0}}{d x^{2}} d x \quad U_{k}=\frac{1}{2} \int_{0}^{l} \rho A u^{2} u_{0}^{2} d x
$$

where $\rho$ is mass density of the beam.

The work done by external forces (potential energy) is defined as

$$
U_{p}=\frac{1}{2}\left[\int_{0}^{l} F\left(\frac{d u_{0}}{d x}\right)^{2} d x+\int_{0}^{l} \rho_{e} u_{0} d x\right]
$$

where $F=F_{m}+F_{\theta}$ is the compressive force applied to the beam. Here, $F_{m}$ and $F_{\theta}$ both are axial forces due to change in mechanical load and temperature, respectively, and $F_{\theta}$ is related with temperature $\theta$ as

$$
F_{\theta}=-\frac{E A \gamma_{x} \theta}{1-2 \nu}
$$

where $\rho_{e}=k_{1} u_{0}-k_{2}\left(d^{2} u_{0} / d x^{2}\right)$ is the elastic medium density and $E A$ is tensile rigidity. Here, $k_{1}$ and $k_{2}$ are Winkler elastic modulus and Pasternak shear elastic modulus, respectively. According to the Winkler elastic modulus, the force on the foundation is directly proportional to deflection while the Pasternak model assumes the existence of shear interaction between the spring elements.

Using equations (2.9) and (2.10) in (2.4) and equating the coefficient of $\delta u_{0}$ to zero, equation (2.4) reduces to

$$
\frac{d^{2} M}{d x^{2}}+\left(F \frac{d^{2}}{d x^{2}}-k_{1}+k_{2} \frac{d^{2}}{d x^{2}}+\rho A \omega^{2}\right) u_{0}=0
$$

The constitutive equations of the nonclassical Euler-Bernoulli beam is given as

$$
-E I \frac{d^{2} u_{0}}{d x^{2}}=M-\xi \frac{d^{2} M}{d x^{2}}
$$

where $E I$ is defined as bending rigidity.

Using (2.12) and (2.13), the bending moment for nonlocal elasticity theory is written as

$$
M=-E I \frac{d^{2} u_{0}}{d x^{2}}+\xi\left(-\rho A \omega^{2} u_{0}-F \frac{d^{2} u_{0}}{d x^{2}}+k_{1} u_{0}-k_{2} \frac{d^{2} u_{0}}{d x^{2}}\right)
$$

Equating the maximum kinetic and potential energy, the dimensionless equation of motion for nonlocal EBT nanobeams is expressed as

$$
\begin{aligned}
& \frac{E I}{l^{2}}\left(1+\widehat{F} \beta^{2}+K_{2} \beta^{2}\right)\left(\frac{d^{2} U}{d X^{2}}\right)^{2}+\frac{E I}{l^{2}}\left(-K_{1} \beta^{2} U-K_{2} U+\alpha^{2} \beta^{2} U\right) \frac{d^{2} U}{d X^{2}} \\
& +\frac{E I}{l^{2}} \widehat{F}\left(\frac{d^{2} U}{d X^{2}}\right)^{2}+\frac{E I}{l^{2}}\left(K_{1}-\alpha^{2}\right) U^{2}=0
\end{aligned}
$$

The dimensionless form of parameters is as follows

$$
\begin{array}{lccc}
X=\frac{x}{l} & U=\frac{u_{0}}{l} \quad & \beta=\frac{e_{0} a}{l} \quad K_{1}=\frac{k_{1} l^{4}}{E I} \quad K_{2}=\frac{k_{2} l^{2}}{E I} \\
\widehat{F}=\frac{F l^{2}}{E I} & \widehat{F}_{m}=\frac{F_{m} l^{2}}{E I} & \widehat{F}_{\theta}=\frac{F_{\theta} l^{2}}{E I} & \alpha^{2}=\frac{\rho A \omega^{2} l^{4}}{E I}
\end{array}
$$




\section{Solution procedure}

To solve equation (2.15), Rayleigh-Ritz method (R-R) is employed with a Legendre's polynomial as a shape function. This method is generally used for finding an approximate solution for different types of mechanical engineering problems and is often used to determine first eigen frequencies and eigenfunctions of continuous linear elastic systems. The approximate solution of eigenvalues and eigenfunctions of the continuous system is improved by using a large number of terms in the Ritz expansion and the error in the approximation of the eigenfunction being measured in a certain norm. The boundary function is chosen in such a way so that it satisfies the essential end conditions of the beam.

According to R-R method, the displacement function can be written as a sum of polynomials as

$$
U(X)=\sum_{n=1}^{N} b_{n} \psi_{n}
$$

where $b_{n}$ are unknowns to be determined, $\psi_{n}$ are orthonormal polynomials and $N$ is the number of terms needed to find the results up to desired level of accuracy. Here we used a Legendre's polynomial as an orthonormal polynomial.

The Legendre polynomials are chosen in such a way that they bijectively map the interval $[0,1]$ to $[1,-1]$. This is to show that the polynomials are orthogonal on $[0,1]$ to satisfy the end conditions $\psi_{n}(0)=\psi_{n}(1)=0, n \geqslant 1$. To fulfill the conditions of orthogonality in the interval $[0,1]$, modified form of the Legendre polynomial is used and is defined as

$$
\psi_{n}(X)=\left[\frac{1}{n !} \frac{d^{n}}{d X^{n}}\left(X^{2}-X\right)^{n}-(-1)^{n}\right](X-1)
$$

Some of the modified Legendre polynomials over the interval $[0,1]$ are as follows

$$
\begin{aligned}
& \psi_{1}(X)=-2 X+2 X^{2} \quad \psi_{2}(X)=6 X-12 X^{2}+6 X^{3} \\
& \psi_{3}(X)=-12 X+42 X^{2}-50 X^{3}+20 X^{4} \\
& \psi_{4}(X)=20 X-110 X^{2}+230 X^{3}-210 X^{4}+70 X^{5} \\
& \psi_{5}(X)=-30 X+240 X^{2}-770 X^{3}+1190 X^{4}-882 X^{5}+252 X^{6}
\end{aligned}
$$

These modified Legendre polynomials can be used as a set of basis functions and satisfy the special properties $\psi_{n}(0)=\psi_{n}(1)=0, n \geqslant 1$ at the boundaries. Putting $\widehat{F}_{m}=-P$ and the inertia term $\alpha^{2}$ to zero in equation (2.15), the following governing equations are obtained to analyze the critical buckling load

$$
\left(1-K_{2} \beta^{2}+\frac{\widehat{F}_{\theta} l^{2}}{E I}+\frac{F_{\theta} l^{2}}{E I} \beta^{2}\right)\left(\frac{d^{2} U}{d X^{2}}\right)^{2}-\left(K_{1} \beta^{2} U+K_{2} U\right) \frac{d^{2} U}{d X^{2}} K_{1} U^{2}=\widehat{P}\left[\left(\frac{d U}{d X}\right)^{2}+\beta^{2}\left(\frac{d^{2} U}{d X^{2}}\right)^{2}\right]
$$

where $\widehat{P}=P l^{2} /(E I)$.

Incorporating equation (3.1) into equation (3.2), solution of equation (3.3) is obtained by minimizing the buckling load with respect to unknown coefficients $b_{j}, j=1,2, \ldots, n$. Thus, by putting $\partial \widehat{P} / \partial b_{j}=0$, the solution of equation (3.3) in matrix form is

$$
\mathbf{S Y}=\widehat{P} \mathbf{B Y}
$$


where $\mathbf{Y}=\left[b_{1}, b_{2}, \ldots, b_{n}\right]^{\mathrm{T}}$ is the transpose matrix of the unknown coefficients $b_{j}, j=1,2, \ldots, n$ and the stiffness and buckling matrices are given by

$$
\begin{aligned}
& S(i, j)=\int_{0}^{1}\left[\left(1+2 K_{2} \beta^{2}+2 \widehat{F} \beta^{2}\right) \ddot{\psi}_{i} \ddot{\psi}_{j}+2 \widehat{F} \beta^{2} \dot{\psi}_{i} \dot{\psi}_{j}\right. \\
& \left.+2 K_{1} \psi_{i} \psi_{j}-\left(K_{2}+K_{1}\right) \ddot{\psi}_{i} \psi_{j}-\left(K_{2}+K_{1}\right) \ddot{\psi}_{i} \ddot{\psi}_{j}\right] d X \\
& B(i, j)=\int_{0}^{1}\left(2 \dot{\psi}_{i} \dot{\psi}_{j}+2 \beta^{2} \ddot{\psi}_{i} \ddot{\psi}_{j}\right) d X
\end{aligned}
$$

where $i, j=1, \ldots, n, \dot{\psi}_{i}$ and $\ddot{\psi}_{i}$ represents derivatives of the first and second order of $\psi_{i}(X)$ with respect to $X$ for all $i$ and $j$. Equation (3.4) is an eigen value problem in which the smallest eigen value will provide the critical buckling load which is to be obtained by equating the determinant of the coefficient matrix to zero. Corresponding eigenvectors are used as shape functions and for finding the critical buckling loads and post buckling behavior of beams and columns.

\section{Results and discussions}

To obtain the numerical results, values of different parameters for a single-walled carbon nanotube according to (Benzair et al., 2008) and (Murmu and Pradhan, 2009) are given in Table 1.

Table 1. Values of different parameters to determine buckling load (Benzair et al., 2008) and (Murmu and Pradhan, 2009)

\begin{tabular}{|l|l|}
\hline \multicolumn{1}{|c|}{ Parameter } & \multicolumn{1}{c|}{ Value } \\
\hline \hline Modulus of elasticity & $1 \mathrm{TPa}$ \\
\hline \multirow{2}{*}{$\begin{array}{l}\text { Thermal expansion } \\
\text { coefficient }\end{array}$} & $-1.4 \cdot 10^{-6}$ for low temperature environment \\
\cline { 2 - 2 } & $1.0 \cdot 10^{-6}$ for high temperature environment \\
\hline Poisson's ratio & 0.19 \\
\hline
\end{tabular}

Values in Table 2 show the critical buckling load for clamped-clamped end conditions for varying values of the aspect ratio and nonlocal scale parameter.

Table 2. Critical buckling load using the present approach and (Pradhan and Reddy, 2011) for the clamped-clamped (C-C) case

\begin{tabular}{|c|c|c|c|c|c|c|c|c|}
\hline \multirow{2}{*}{$\begin{array}{c}\text { Aspect } \\
\text { ratio } \\
(l / d)\end{array}$} & \multicolumn{3}{|c|}{$\begin{array}{c}\text { Nonlocal parameter }\left[\mathrm{nm}^{2}\right] \\
\text { Pradhan and Reddy }(2011)\end{array}$} & \multicolumn{4}{c|}{$\begin{array}{c}\text { Nonlocal parameter }\left[\mathrm{nm}^{2}\right] \\
\text { Present approach }\end{array}$} \\
\cline { 2 - 9 } & $\xi=0$ & $\xi=1.0$ & $\xi=1.5$ & $\xi=2.0$ & $\xi=0$ & $\xi=1.0$ & $\xi=1.5$ & $\xi=2.0$ \\
\hline \hline 10 & 9.6311 & 8.2298 & 6.3549 & 3.2367 & 9.6318 & 8.2314 & 6.3512 & 3.2369 \\
\hline 12 & 5.3601 & 4.3316 & 3.3686 & 2.1855 & 5.3617 & 4.3325 & 3.3698 & 2.1865 \\
\hline 14 & 3.1114 & 2.6010 & 2.1238 & 1.6296 & 3.1134 & 2.6025 & 2.1230 & 1.6362 \\
\hline 16 & 2.1183 & 1.7992 & 1.5629 & 1.2102 & 2.1193 & 1.8001 & 1.5632 & 1.2050 \\
\hline 18 & 1.7741 & 1.5201 & 1.2251 & 1.0135 & 1.7721 & 1.5124 & 1.2253 & 1.0090 \\
\hline 20 & 1.6125 & 1.3459 & 1.1248 & 0.9905 & 1.6134 & 1.3462 & 1.1253 & 0.9963 \\
\hline
\end{tabular}

It is observed from the numerical results that the critical buckling load decreases as the length to diameter ratio increases, and this effect is more significant for higher values of the nonclassical scale parameter. Figure 1a shows variation in the critical buckling strain (or load) 
with the length-to-diameter ratio for $\mathrm{C}-\mathrm{C}$ end conditions. Multiple graphs are plotted for different nonclassical parameter values.

It is to be noted that if the nonclassical parameter is neglected, the obtained results correspond to those that are obtained from the local theory. It is also observed from the graph that in order to set a lower buckling load, the value of the scale coefficient is to be increased. These observations are verified with (Pradhan and Reddy, 2011).

(a)

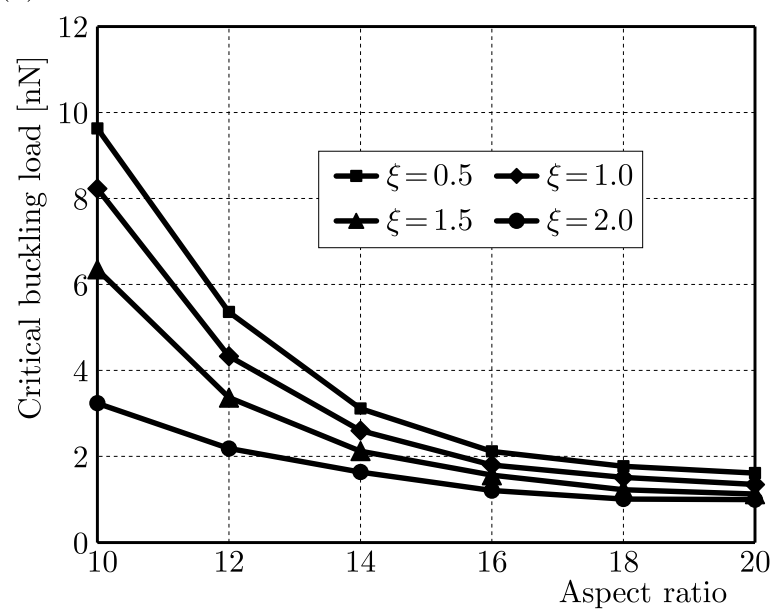

(b)

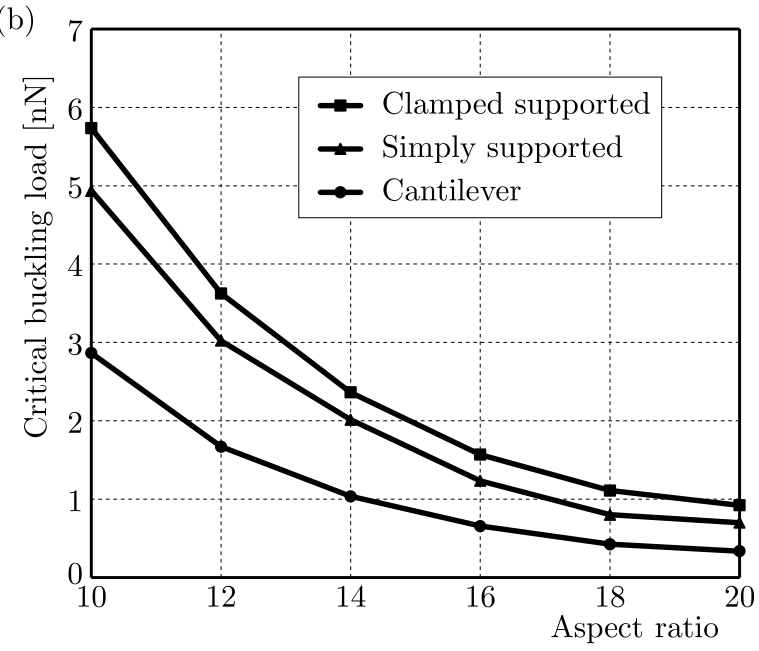

Fig. 1. (a) Variation in the critical buckling load with different values of the aspect ratio. (b) Impact of the aspect ratio on the critical buckling load

Figure 1b illustrates the impact of the length-to-diameter ratio on the lowest (critical) buckling load for various boundary conditions. The load value decreases for all boundary conditions as the aspect ratio increases. Moreover, clamped-simply supported end conditions provide a higher buckling load as compared to simply supported and cantilever beams. The graph shows that buckling solutions are highly influenced by the small scale coefficient. Buckling strain is high for a lower aspect ratio. The obtained results are in agreement with (Wang C.M. et al., 2006).
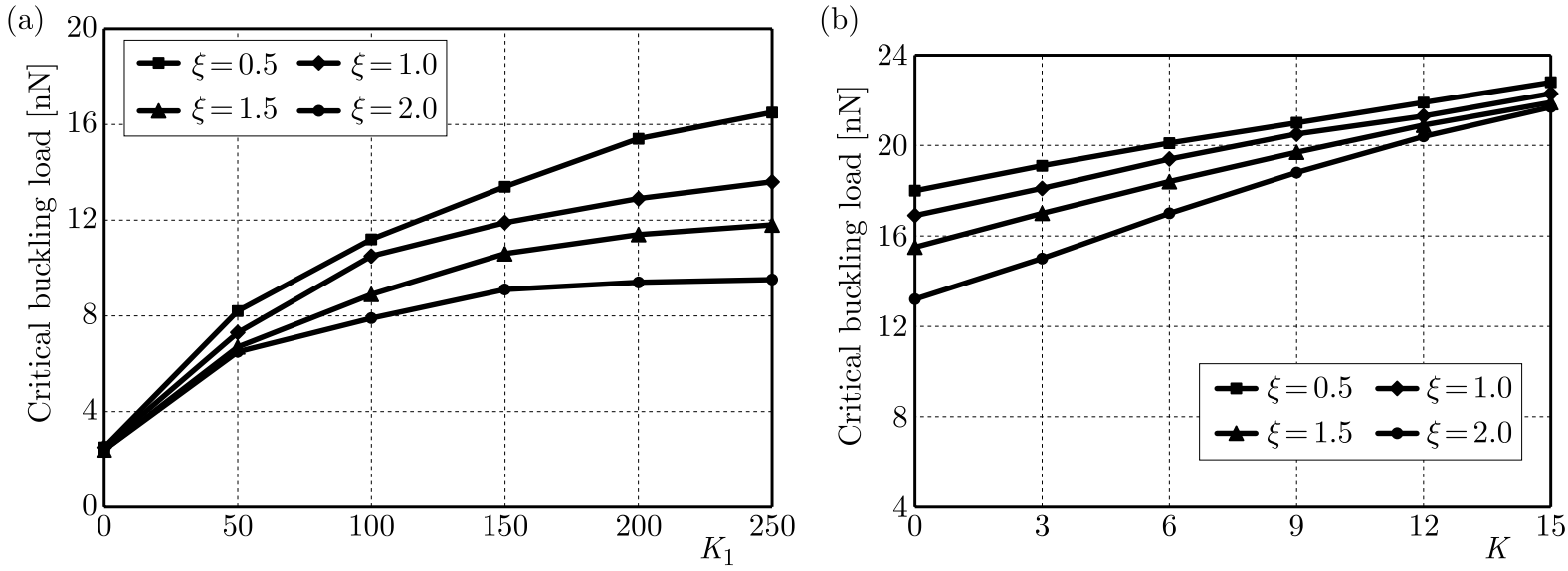

Fig. 2. (a) Distribution of the critical buckling load for different Winkler elastic moduli. (b) Impact of the Pasternak shear elastic modulus on the critical buckling load

Figure 2a represents the impact of the Winkler elastic constant. To study this effect, different physical values are taken as $\theta=20, l / d=15$ and $K_{2}=0$. Variations in the buckling load are shown for different scale coefficients, and the Winkler modulus is taken between 0-250. It is shown in the graph that the critical buckling load increases with the increment in the Winkler 
modulus. For higher values of the scale coefficient, nonlinear behavior of the lowest buckling load for different values of the Winkler elastic medium is observed. The reason behind this effect is that the nanotube becomes rigid after increasing the value of the Winkler elastic medium constant.

Figure $2 \mathrm{~b}$ demonstrates the change in the critical buckling load parameter with respect to the Pasternak shear modulus parameter for various values of nonlocal parameters. Different parameters are taken as $\theta=20, l / d=15$ and $K_{1}=0$ to study the effect of the elastic medium on buckling solutions. It is noticed that the increasing of the Pasternak elastic modulus increases the critical buckling load, and this increment is linear in nature due to the commanding nature of this foundation. It can be easily seen that the critical buckling loads in the Pasternak model are larger than in the Winkler model. The critical buckling loads of single walled carbon nanotubes for higher values of small scale coefficients are small compared to lower values of the scale coefficient. The obtained results are in validation with (Murmu and Pradhan, 2009).

Figure 3 shows the effect of temperature on the critical buckling load for two different environments (Murmu and Pradhan, 2010) and $K_{2}=3$. It is observed from the graph that if the nanobeam is placed in low temperature environment, the critical buckling strain increases as temperature increases. However, in high temperature environment, the critical buckling strain decreases with an increase in temperature. This is due to the fact that rigidity of the nanobeam in low temperature environment increases as temperature increases, and in high temperature environment the rigidity of a single walled carbon nanotube decreases as temperature increases. Similar interpretations were also presented in (Murmu and Pradhan, 2010) and (Chakraverty and Behera, 2015).

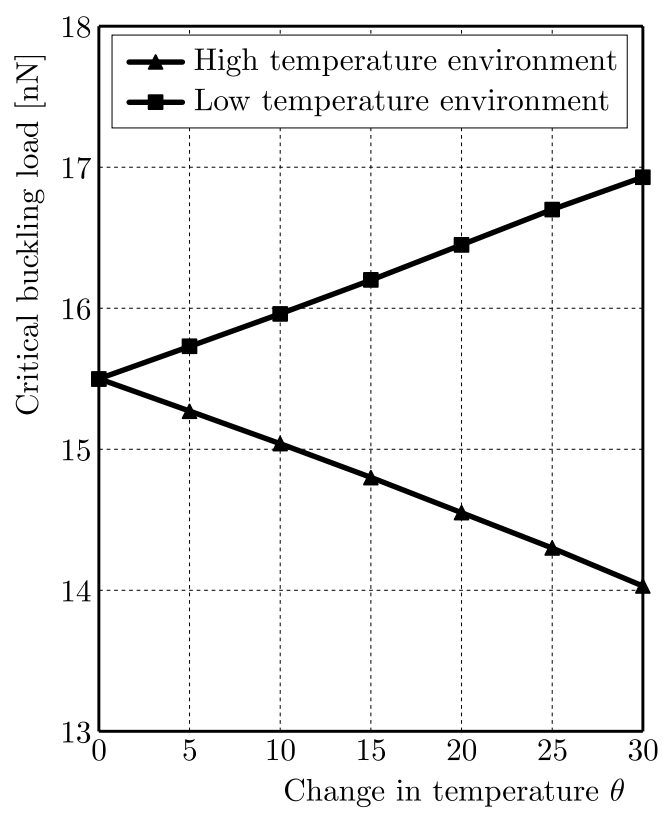

Fig. 3. Variation in the critical buckling load due to different temperature environments

\section{Conclusion}

In the present paper, considering the effect of temperature in conjunction with two foundation models, the mechanical analysis of single walled carbon nanotubes is studied. Legendre's polynomial is used as a characteristic polynomial along with the Rayleigh-Ritz method playing a significant contribution to this study. The orthogonality of these polynomials ensures the results to be found valid with a reasonable accuracy. For two-parameter models, the influence of phy- 
sical parameters like the elastic coefficients, nonlocal parameter, aspect ratio and temperature is discussed graphically. Conclusions are drawn with the help of the obtained numerical results and are in fair agreement with those reported in literature. It is found that the critical buckling load increases as the nonlocal parameter increases. Moreover, it is also observed that in the case of the Pasternak foundation, the critical buckling load behaves linearly while for the Winkler foundation this behavior is nonlinear. Critical buckling loads behave nonlinearly for the Winkler elastic modulus because single walled carbon nanotubes become stiffer after an increment in the Winkler elastic modulus. The reason for linearity may be due to the commanding nature of the Pasternak type elastic foundation.

\section{References}

1. Ansari R., Gholami R., Darabi M.A., 2011, Thermal buckling analysis of embedded singlewalled carbon nanotubes with arbitrary boundary conditions using the nonlocal Timoshenko beam theory, Journal of Thermal Stresses, 34, 12, 1271-1281

2. Benzair A., Tounsi A., Besseghier A., Heireche H., Moulay N., Boumia L., 2008, The thermal effect on vibration of single-walled carbon nanotubes using nonlocal Timoshenko beam theory, Journal of Physics D: Applied Physics, 41, 22, 225404

3. Chakraverty S., Behera L., 2015, Vibration and buckling analyses of nanobeams embedded in an elastic medium, Chinese Physics B, 24, 9, 097305

4. Challamel N., 2011, Higher-order shear beam theories and enriched continuum, Mechanics Research Communications, 38, 5, 388-392

5. Dai H., Hafner J.H., Rinzler A.G., Colbert D.T., Smalley R.E., 1996, Nanotubes as nanoprobes in scanning probe microscopy, Nature, 384, 6605, 147-150

6. ERINGEn A.C., 1972, Linear theory of nonlocal elasticity and dispersion of plane waves, International Journal of Engineering Science, 10, 5, 425-435

7. ERINGen A.C., 1983, On differential equations of nonlocal elasticity and solutions of screw dislocation and surface waves, Journal of Applied Physics, 54, 9, 4703

8. Eringen A.C., 2002, Nonlocal Continuum Field Theories, Springer Science \& Business Media

9. Iıנrma S., 1991, Helical microtubules of graphitic carbon, Nature, 354, 6348, 56-58

10. Kim P., Lieber C.M., 1999, Nanotube nanotweezers, Science, 286, 5447, 2148-2150

11. Li X., Bhushan B., Takashima K., Baek C.W., Kim Y.K., 2003, Mechanical characterization of micro/nanoscale structures for MEMS/NEMS applications using nanoindentation techniques, Ultramicroscopy, 97, 1-4, 481-494

12. Lu Q., Bhattacharya B., 2005, The role of atomistic simulations in probing the small-scale aspects of fracture - a case study on a single-walled carbon nanotube, Engineering Fracture Mechanics, 72, 13, 2037-2071

13. Murmu T., Pradhan S.C., 2009, Buckling analysis of a single-walled carbon nanotube embedded in an elastic medium based on nonlocal elasticity and Timoshenko beam theory and using DQM, Physica E: Low-Dimensional Systems and Nanostructures, 41, 7, 1232-1239

14. Murmu T., Pradhan S.C., 2010, Thermal effects on the stability of embedded carbon nanotubes, Computational Materials Science, 47, 3, 721-726

15. Nagar P., Tiwari P., 2017, Recursive differentiation method to study the nature of carbon nanobeams: A numerical approach, AIP Conference Proceedings, 1897, 1, 020009, AIP Publishing

16. NeJAD Z.M., Hadi A., 2016, Eringen's non-local elasticity theory for bending analysis of bidirectional functionally graded Euler-Bernoulli nano-beams, International Journal of Engineering Science, 106, 1-9 
17. Norouzzadeh A., Ansari R., 2017, Finite element analysis of nano-scale Timoshenko beams using the integral model of nonlocal elasticity, Physica E: Low-Dimensional Systems and Nanostructures, 88, 194-200

18. Peddieson J., Buchanan G.R., McNitt R.P., 2003, Application of nonlocal continuum models to nanotechnology, International Journal of Engineering Science, 41, 3, 305-312

19. Pradhan S.C., Reddy G.K., 2011, Buckling analysis of single walled carbon nanotube on Winkler foundation using nonlocal elasticity theory and DTM, Computational Materials Science, 50, 3, 1052-1056

20. Rafiee R., Moghadam R.M., 2014, On the modeling of carbon nanotubes: A critical review, Composites Part B: Engineering, 56, 435-449

21. Reddy J.N., El-Borgi S., 2014, Eringen's nonlocal theories of beams accounting for moderate rotations, International Journal of Engineering Science, 82, 159-177

22. Sakharova N.A., Antunes J.M., Pereira A.F.G., Fernandes J.V., 2017, Developments in the evaluation of elastic properties of carbon nanotubes and their heterojunctions by numerical simulation, AIMS Materials Science, 4, 3, 706-737

23. Semmah A., Tounsi A., Zidour M., Heireche H., Naceri M., 2015, Effect of the chirality on critical buckling temperature of zigzag single-walled carbon nanotubes using the nonlocal continuum theory, Fullerenes, Nanotubes and Carbon Nanostructures, 23, 6, 518-522

24. Wang C.M., Zhang Y.Y., Ramesh S.S., Kitipornchai S., 2006, Buckling analysis of microand nano-rods/tubes based on nonlocal Timoshenko beam theory, Journal of Physics D: Applied Physics, 39, 17, 3904

25. Wang Q., Varadan V.K., QueK S.T., 2006, Small scale effect on elastic buckling of carbon nanotubes with nonlocal continuum models, Physics Letters A, 357, 2, 130-135

Manuscript received November 6, 2017; accepted for print May 28, 2018 